\section{An Apparent Failure of the Hund Theory.}

The Hund theory of spectra includes, as an essential part of it, a prediction of the individual levels of an ion to which component term sequences will converge. The evidence supporting the predictions seem reasonably good in cases of spectra the character of which is determined by less than half-filled electron groups. On the other hand, the following two items of evidence seem to indicate that the theoretical predictions are not fulfilled in spectra determined by nearly completed electron groups.

1. The Paschen $s$ terms in Ne I are, without doubt, the ${ }^{3} P$ and ${ }^{1} P$, which arise from an electron structure $2 p^{5} n s(n \geqslant 3)$. The Is terms can be arranged unambiguously from both position and Zeeman effect as follows :

$$
{ }^{3} P_{2}=1 s_{5},{ }^{3} P_{1}=1 s_{4},{ }^{3} P_{0}=1 s_{3},{ }^{1} P_{1}=1 s_{2} .
$$

Consequently, if one accepts Paschen's series, ${ }^{3} P_{2}$ and ${ }^{3} P_{1}$ converge to one limit; and ${ }^{3} P_{0}$ and ${ }^{1} P_{i}$ to a higher limit, the two limits together being the ${ }^{2} P\left(p^{5}\right)$ of Ne II. This is in definite disagreement with the theory, which would reverse the limits of ${ }^{3} P_{1}$ and ${ }^{1} P_{1}$. Since the nature of the terms is determined empirically in this case by the first members only and the limits by the higher members, it is possible to reconcile observation and theory, as Hund has done, by associating $1 s_{4}$ with the $s_{2}$ series as its first member; and $1 s_{2}$ in the same way with the $s_{4}$ series. But there is now evidence against such a change. The series of $s_{2}$ and $s_{4}$ terms combine with the lowest term of the spectrum ${ }^{1} S_{0}\left(2 p^{6}\right)$ to give a far ultra - violet series of pairs ${ }^{1} S_{0}-n s_{2}$, ${ }^{1} S_{0}-n s_{4}$. In this series the line ${ }^{1} S_{0}-n s_{2}$ is the stronger of each of the pairs. This statement is based not only on the published material, but also on personal observation of plates taken in this laboratory. Such a regularity of intensities is incompatible with Hund's distortion of the series and leads definitely to the conclusion that the theoretical prediction is not satisfied for such sequences as the $s$-terms of neon. The whole argument applies equally to the corresponding terms of argon.

2 . The second item of evidence is contained in the spectra of atoms and ions, of which the structure is based on $9 d$-electrons. Ni I, Cu II, Pd I, Ag II all show sequences of ${ }^{3} D,{ }^{1} D$ terms of origin $d^{9} s$ converging to the ${ }^{2} D\left(d^{9}\right)$ of the higher ion. In $\mathrm{Cu}$ II, $\mathrm{Ni}$, and Ag II only two series members are known, but they are well verified in every case by intensities and in $\mathrm{Cu}$ II also by Zeeman effects. In all cases the calculated limits of ${ }^{3} D_{3}$ and ${ }^{3} D_{2}$ fall close together, as do those of ${ }^{3} D_{1}$ and ${ }^{1} D_{2}$, contrary to the theory, which predicts coincidence of the limits in pairs ${ }^{3} D_{3},{ }^{1} D_{2}$; ${ }^{3} D_{2},{ }^{3} D_{1}$. In Pd I the evidence is more striking, since three series members are present. To pick them correctly, it is necessary to identify consistently all the terms of the spectrum from intensities. This leads to an identification of the middle set of terms exactly as given by Bechert and Catalan and in disagreement with McLennan and Smith. The second member of the $d^{9} s$ sequence is then McLennan's with ${ }^{3} D_{2}$ and ${ }^{1} D_{2}$ interchanged. In the third member, this interchange is again necessary, and also the addition of McLennan's ${ }^{3} G_{4}$ as ${ }^{3} D_{3}$. These identifications are unambiguous. The limits calculated from a Ritz series formula lead to the same disagreement with theory as the similar two term series. The separation of the two limits ${ }^{2} D_{3}$ and ${ }^{2} D_{2}$ of $\mathrm{Pd} \mathrm{II}$, is so great (more than 3500 wavenumbers) that there can scarcely be any doubt of the reality of the coincidences of the real limits even though the calculated limits differ by 80 units in one case $\left({ }^{3} D_{3}\right.$ and $\left.{ }^{3} D_{2}\right)$ and 20 units in the other $\left({ }^{3} D_{1}\right.$ and $\left.{ }^{1} D_{2}\right)$.

The disagreements with the Hund theory here pointed out are the only ones of which I am aware.
More evidence is certainly advisable and it is being sought in this laboratory.

I cannot offer any explanation of these apparent failures of the theory. It seems that the operations with the electron vectors which lead to the predictions may be carried out in a great variety of ways and lead always to the same result so long as they are treated consistently in both series and limit terms.

Palmer Physical Laboratory,

A. G. Shenstone.

Princeton University,

Princeton, New Jersey, Feb. 16.

\section{A Change of Wave-length in Light Scattering.}

Further observations by Mr. Krishnan and myself on the new kind of light-scattering discovered by us have been made and have led to some very surprising and interesting results.

In order to convince ourselves that the secondary radiation observed by us was a true scattering and not a fluorescence, we proceeded to examine the effect in greater detail. The principal difficulty in observing the effect with gases and vapours was its excessive feebleness. In the case of substances of sufficient light-scattering power, this difficulty was overcome by using an enclosed bulb and heating it up so as to secure an adequate density of vapour. Using a blueviolet filter in the track of the incident light, and a complementary green-yellow filter in front of the observer's eye, the modified scattered radiation was observed with a number of organic vapours, and it was even possible to determine its state of polarisation. It was found that in certain cases, for example, pentane, it was strongly polarised, while in others, as for example naphthalene, it was only feebly so, the behaviour being parallel to that observed in the liquid state. Liquid carbon dioxide in a steel observation vessel was studied, and exhibited the modified scattering to a notable extent. When a cloud was formed within the vessel by expansion, the modified scattering brightened up at the same time as the ordinary or classical scattering. The conclusion is thus reached that the radiations of altered wave-length from neighbouring molecules are coherent with each other.

A greater surprise was provided by the spectroscopic observations. Using sunlight with a blue filter as the illuminant, the modified scattered radiation was readily detected by the appearance in the spectrum of the scattered light of radiations absent from the incident light. With a suitably chosen filter in the incident light, the classical and modified scatterings appeared as separate regions in the spectrum separated by a dark region. This encouraged us to use a mercury arc as the source of light, all radiations of longer wave-length than $4358 \mathrm{~A}$. being cut out by a filter. The scattered radiations when examined with a spectroscope showed some sharp bright lines additional to those present in the incident light, their wave-length being longer than $4358 \mathrm{~A}$.; at least two such lines were prominent and appeared to be accompanied by some fainter lines, and in addition a continuous spectrum. The relation of frequencies between the new lines and those present in the incident light is being investigated by photographing and measuring the spectra. The preliminary visual observations appear to indicate that the position of the principal modified lines is the same for all substances, though their intensity and that of the continuous spectrum does vary with their chemical nature.

210 Bowbazar Street,

C. V. Raman. Calcutta, Mar. 8.

No. 3051, VoL. 121] 